\title{
An $a b$ initio Study of a Model of Single Wall GaN Nanotubes with Oxygen and Zinc as Impurities: Structural and Electronic Properties
}

\author{
K. Rezouali and M.A. Belkhir* \\ University of Bejaia, Department of Physics \\ Theoretical Physics Laboratory, Solid State Physics Group \\ Bejaia 06000, Algeria \\ (Received August 24, 2007; revised version December 24, 2007)
}

\begin{abstract}
We report a comprehensive theoretical study of structural and electronic properties of substitutional oxygen and zinc contaminations in a model of single wall GaN nanotubes by means of $a b$ initio supercell calculations. Our investigation yields many interesting results. The following ones deserve to be developed. Oxygen forms a shallow donor in the single wall GaN nanotubes as in bulk GaN polytypes. Its ionization energy is deeper than its counterpart in the bulk wurtzite GaN, so it can be a suitable $n$-type dopant in high temperature applications. Zinc is a relatively shallow acceptor at gallium site. It behaves as charge carrier trap at nitrogen site. The site preference of zinc impurity depends on the stoichiometry. Zinc at gallium site has small ionization energy, thus it would be an efficient $p$-dopant in GaN nanotubes unlike in bulk GaN polytypes.
\end{abstract}

PACS numbers: 61.46.Fg, 61.72.Bb, 73.61.Ey, 73.61.Wp

\section{Introduction}

The performance improvements of gallium nitride $(\mathrm{GaN})$ device applications depend strongly on the features of intrinsic and extrinsic defects in the compound. Bulk GaN has long been viewed as a promising material for lighting device applications. Oxygen is a common contamination in bulk GaN polytypes. Zinc is the impurity material most widely used in $p$-doping of GaN. Oxygen favors the nitrogen site and acts as a donor making $n$-type conductivity. Zinc substitutes both gallium and nitrogen sites forming acceptors.

What makes GaN popular is not only it is more robust but it can emit in the short wave-length part of the visible spectrum. For example, there are now GaN-based high-efficiency blue and green light-emitting diodes [1].

*corresponding author; e-mail: belkhir_a@yahoo.fr 
And just when researchers thought things could not get better for GaN, a whole new field of research has opened up in the form of GaN nanotubes [2]. These nanotubes were synthesized by an "epitaxial casting" strategy by the research group of Peidong Yang at Lawrence Berkeley National Laboratory. ZnO nanowires, grown on sapphire wafers, were used as templates for epitaxial overgrowth of thin GaN layers in a chemical vapor deposition (CVD) system. The result of electron diffraction measurements showed that the nanotubes obtained in this way are single-crystal, and in this respect they are fundamentally different from theoretically simulated GaN nanotubes [3] with the conventional tubular forms of carbon atoms [4]. These nanotubes were studied by transmission electron microscopy, which showed that they have inner diameters of 30-200 nm and wall thicknesses of 5-50 nm. X-ray diffraction measurement showed that they are wurtzite structure and oriented along their $c$-axis was revealed, although a few atoms of zinc and oxygen had been incorporated into the GaN tube wall. These hollow nanotubes are robust, electrically and optically active. They could therefore open up an exciting field in nanofluidic biochemical sensing, nanoscale electronics, and optoelectronics.

It is well known that the performance improvements of these promising device applications depend strongly on the features of intrinsic and extrinsic defects in these nitride compounds. Therefore, knowing the accurate position of the donor and acceptor levels of these systems is an issue of great importance in the understanding of optical properties and practical applications of these nanotubes. The substitutional impurities are well known to have a major influence on the electronic properties of nanotubes. Many papers have been published regarding the effects of subtitutional atoms on the structure and electronic properties of nanotubes. For example, the structure and electronic properties of substitutional nitrogen and boron impurities have been studied in silicon carbide ( $\mathrm{SiC}$ ) nanotubes [5]. It was found that nitrogen is an $n$-type dopant or it is a charge carrier trap depending on the width of the band gap of $\mathrm{SiC}$ nanotubes while boron can form a shallow and relatively deep acceptor depending on the stoichiometry of $\mathrm{SiC}$ nanotubes. The manipulation of the Fermi level in SiC nanotubes by controlling the concentration of nitrogen, the concentration and the site selection of boron impurities can be an important issue in biological applications and in chemical sensors.

Up to now, the structure and electronic properties of substitutional impurities in GaN nanotubes have not yet been reported. Considering the potential applications of GaN nanotubes, the studies in this aspect are desirable. In this paper, we report the density functional calculations that investigate the electronic properties of these nanotubes, and how those are modified in the presence of oxygen and zinc impurities. To accomplish this task, we choose a single-crystal GaN nanotube having one atomic layer as a prototype. This nanotube has been generated from an ideal wurtzite GaN crystalline structure and is therefore fundamentally different from the tube structures which can be viewed as the mapping of graphitic 
(a)

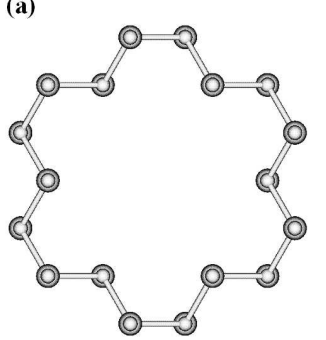

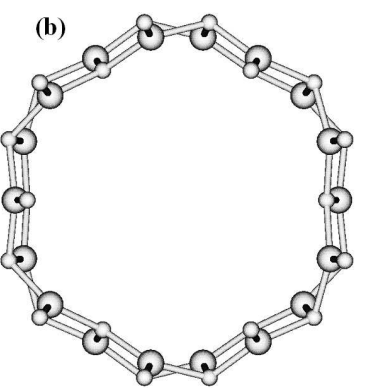

Fig. 1. Initial (a) and optimized (b) GaN SWNT supercell. The large spheres are Ga atoms and the smaller spheres are $\mathrm{N}$ atoms.

sheet of GaN onto the surface of a cylinder. The nanotube modelled in this way has a non-circular cross-section (Fig. 1a). To refer to our GaN nanotube, we will use the same terminology as for carbon nanotubes and will talk about GaN single walled nanotube (GaN SWNT). Even if GaN nanotubes with single wall have not been synthesized yet, the predictions of the present work are important for further efforts to achieve. Our results showed that oxygen forms a shallow donor in GaN SWNT like in bulk GaN polytypes. Its ionization energy is deeper than its counterpart in the bulk wurtzite GaN. Therefore, it can be a suitable $n$-type dopant in high temperature uses for GaN SWNT. Zinc is a relatively shallow acceptor or charge carrier trap depending on the stoichiometry of GaN nanotubes. Zinc at gallium site have small ionization energy, thus it would be an efficient $p$-dopant in GaN nanotubes unlike in bulk GaN polytypes.

\section{Method of calculation}

We have performed our calculations using the SIESTA code [6] package, which implements density functional theory (DFT) with the pseudopotential approximation and a basis set of linear combination of atomic orbitals. We applied the generalized gradient approximation (GGA) for the exchange-correlation functional as parameterized by Perdew, Burke, and Ernzerhof (PBE) [7]. To take into account the effect of core electrons, the pseudopotentials were generated according to the Troullier-Martins scheme [8]. The basis set was double zeta plus polarization orbitals. To compress the basis set, we used an energy cut-off of 0.02 Ry. The mesh cut-off, an energy which corresponds to the grid spacing, was chosen to be $90 \mathrm{Ry}$. First, the lattice vector and the coordinates of atoms were optimized in a GaN SWNT supercell of 36 atoms. This was generated from an ideal wurtzite GaN crystalline structure having experimental lattice parameters $a=3.189 \AA, c=5.185 \AA$. The nanotube supercell contains 18 atoms of Ga and 18 atoms of $\mathrm{N}$. This nanotube has a polygon periphery and extends to infinity along the [0001] direction through the supercell repetition. Viewed with the central tube axis, the diameters of the inner shell and the outer shell are $8.807 \AA$ and $9.764 \AA$, 
respectively (Fig. 1a). Further details can be found elsewhere [9]. We have used a large $(26.53 \AA$ ) vacuum in the lateral directions (the [1010] and the [011̄0] directions) to make sure that the additional interactions which can be caused by the artificial tube-tube interaction are subsequently avoided. The energy of this configuration is minimized without any symmetry constraint using the conjugategradient algorithm, until the residual forces were smaller than $0.001 \mathrm{eV} / \AA$. We used 5 Monkhorst-Pack (MP) special $k$ points [10] along the tube axis for the Brillouin zone summations.

In order to investigate the defects, we used a larger supercell of 108 atoms containing 54 atoms of $\mathrm{Ga}$ and the same number of $\mathrm{N}$ atoms. This was generated following the same procedure as discussed above. This supercell contains 3 layers along the tube axis (the [0001] direction), such that the 36-atoms supercell forms its unit cell. Due to the large size of these defective supercells, only a set of two MP $k$ points along the tube axis were used for the Brillouin zone sampling. All the atoms in these supercells are also allowed to relax without any constraint until the atomic forces were below $0.001 \mathrm{eV} / \AA$.

\section{Results}

\subsection{Perfect GaN SWNT}

We first optimized the GaN SWNT by allowing all the atoms in the supercell to relax without any symmetry constraint. After the relaxation, the puckered cross section of the initial GaN SWNT in Fig. 1a was transformed into a pseudohexagon (see Fig. 1b). It is interesting to note that the tubular morphology of this nanotube is fundamentally different from the usual tubular forms of carbon and boron nitride (BN) nanotubes. In the outer shell, the more electronegative atoms ( $\mathrm{N}$ atoms) are pushed radially outward and the more electropositive atoms (Ga atoms) are displaced inward. In the inner shell, the opposite phenomenon occurs and the Ga atoms move outward while the $\mathrm{N}$ atoms move inward. This result for GaN nanotubes is expected since the overlap between the Ga's and N's pz orbitals is weaker [11]. The relaxed Ga-N bond length is about $1.901 \AA$ which is contracted by $-4.9 \%$ compared to the ideal bond length of $1.95 \AA$ in the bulk. The average nearest $\mathrm{Ga}-\mathrm{Ga}$ and $\mathrm{N}-\mathrm{N}$ distances are $3.292 \AA$ and $3.453 \AA$. According to the Mulliken population analysis, a considerable amount of electron charge $(0.65 e)$ is transferred from Ga to $\mathrm{N}$, resulting in an ionic bonding nature. $\mathrm{N}$ loses some $s$ electrons and gains more $p$ electrons, whereas Ga loses more $p$ than $s$ electrons. The calculated band structure along $\Gamma$ to $X$ indicates clearly that this nanotube has semiconducting feature. Figure 2 shows the band structure along the tube axis ([0001] direction) of the GaN SWNT. X band edge corresponds to the symmetry point $(0,0,1 / 2) 2 \pi / c$. We obtained an indirect semiconductor, where the valenceband maximum (VBM) is located at $0.36 \Gamma X$ and the conduction band minimum (CBM) is at the $\Gamma$ point. The calculated indirect GGA band gap is found to be $2.35 \mathrm{eV}$ which is comparable to the value of $2.31 \mathrm{eV}$ reported by Wang et al. [9]. 


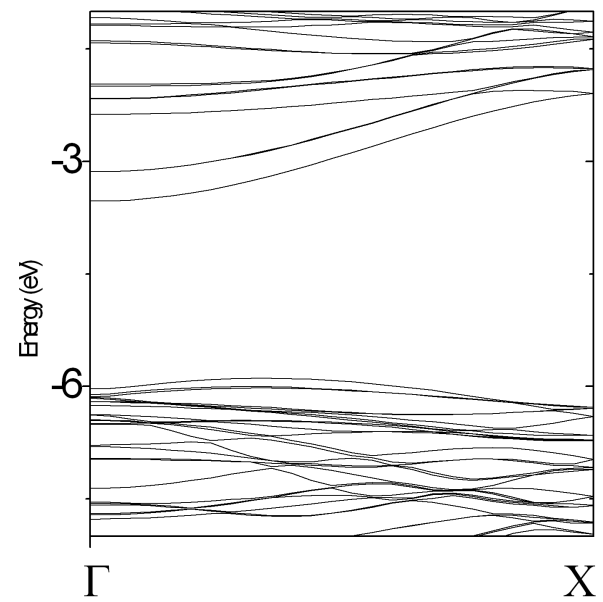

Fig. 2. Band structure along the tube axis of GaN SWNT. $X$ corresponds to the symmetry point $(0,0,1 / 2) 2 \pi / c$ where $c$ is the lattice constant of the perfect nanotube.

To test the stability of the nanotube, we calculated its heat of formation and binding energy. The heat of formation of the GaN SWNT can be calculated as follows:

$$
\Delta H_{\mathrm{f}}=\mu_{\mathrm{GaN}(\mathrm{SWNT})}-\left(\mu_{\mathrm{Ga}(\mathrm{bulk})}\right)+\mu_{\mathrm{N}}
$$

$\mu_{\mathrm{GaN}(\mathrm{SWNT})}$ is the chemical potential of GaN SWNT, which is defined as the total energy per its $\mathrm{Ga}-\mathrm{N}$ unit. $\mu_{\mathrm{Ga}(\mathrm{bulk})}$ is the chemical potential of an orthorhombic bulk gallium. We have taken this value as a reference for the Ga-rich conditions. The chemical potential of $\mathrm{N}$ is taken as the half calculated total energy of $\mathrm{N}_{2}$ molecule. The heat of formation of GaN SWNT is then calculated to be -0.364 $\mathrm{eV}$. For wurtzite bulk GaN, our calculated heat of formation is about $-1.119 \mathrm{eV}$ in good agreement with the value of $-1.14 \mathrm{eV}$ obtained from enthalpy measurements [12]. Therefore, the GaN SWNT is energetically unfavorable over the wurtzite bulk GaN. Yet the small magnitude opens the possibility of forming GaN SWNT as metastable phase. The calculated binding energy of GaN SWNT is about $-5.548 \mathrm{eV}$ /atom. This value is slightly higher than that of zinc blende (ZB) GaN reported in Ref. [3]. This again ensures the possibility of GaN SWNT formation.

\subsection{Oxygen substitutional in GaN SWNT}

Oxygen is a very common unintentional impurity in bulk GaN which enters the material during growth. It inclusively favors a substitutional site $O_{N}$ [13]. The reason for this strong preference is because $\mathrm{O}$ has a size very similar to $\mathrm{N}$. Therefore $\mathrm{O}$ is a single donor which might attract acceptor to reduce the Coulomb energy. This impurity has also been detected in GaN nanotubes [2]. Since the situation is similar in GaN nanotubes, we consider only $\mathrm{O}_{\mathrm{N}}$ in this work. Since it is not clear if the initial polygon structure of the nanotube (Fig. 1a) would change to the SWNT, once O is doped, we begin our calculations with the initial 

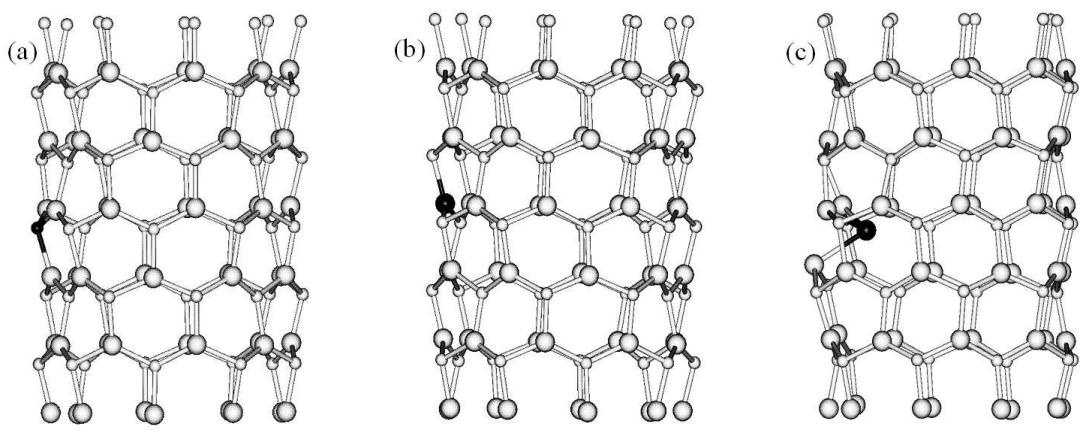

Fig. 3. Optimized geometry of (a) $\mathrm{O}_{\mathrm{N}}$, (b) $\mathrm{Zn}_{\mathrm{Ga}}$ and (c) $\mathrm{Zn}_{\mathrm{N}}$ in GaN SWNT. The light balls represent gallium (larger ones) and nitrogen (smaller ones). The dark balls indicate $\mathrm{O}$ atom (a) and $\mathrm{Zn}$ atom (b) and (c).

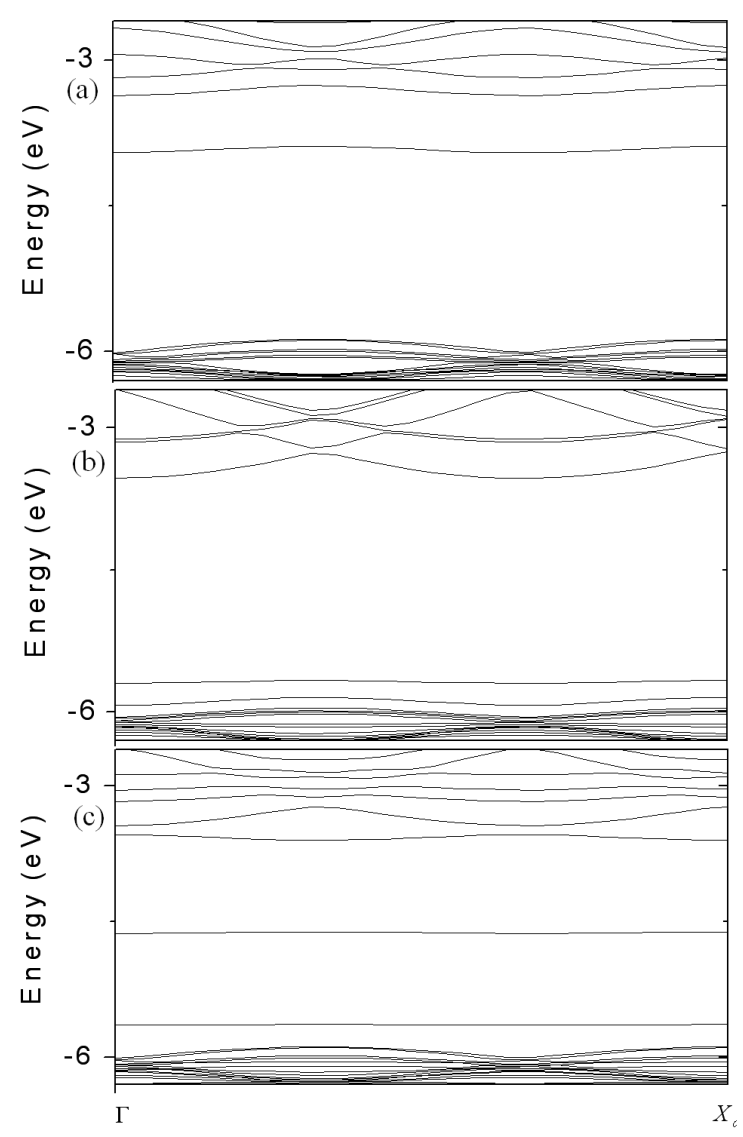

Fig. 4. Band structure along the tube axis of the (a) $\mathrm{O}_{\mathrm{N}}$, (b) $\mathrm{Zn}_{\mathrm{Ga}}$, and (c) $\mathrm{Zn}_{\mathrm{N}}$ in the GaN SWNT supercell. $X_{\mathrm{d}}$ band edge corresponds to the symmetry point $(0,0, q) 2 \pi / L$, where $L$ is the lattice constant of the defective supercell. 
nanotube geometry. To accomplish this task, we just replaced one $\mathrm{N}$ atom by one $\mathrm{O}$ atom in the tube wall. There are two ways the substitution can be achieved depending on the shell where the subtitutional $\mathrm{N}$ site is localized. These two kinds of configurations are: (i) $\mathrm{N}$ site resides in the outer shell (configuration O-out); (ii) $\mathrm{N}$ site resides in the inner shell (configuration O-in). Total energy calculations with geometry optimization have been carried out for these two configurations in order to determine the preferred ground state. It was found that configuration O-out is the favorable configuration, i.e. the ground state configuration. The configuration $\mathrm{O}$-in is $0.019 \mathrm{eV}$ higher in energy. In the ground state configuration, we found that $\mathrm{O}$ tends to relax outward in GaN SWNT. This effect is more pronounced than for $\mathrm{N}$ in the perfect GaN SWNT since the electronegativity difference is even larger. The average $\mathrm{Ga}-\mathrm{O}$ bond length is calculated to be $1.991 \AA$, which is slightly larger than the Ga-N bond length in bulk GaN (1.95 $\AA$ ). The optimized geometry is shown in Fig. 3a. Mulliken charge analysis shows an excessive charge on $\mathrm{O}$ with slightly upper value as compared to $\mathrm{N}$ atoms in the host lattice. $\mathrm{O}_{\mathrm{N}}$ is a single donor in GaN SWNT like in bulk GaN. The occupied shallow donor is at about $0.42 \mathrm{eV}$ below the conduction band edge, i.e. at $E_{\mathrm{V}}+1.93 \mathrm{eV}$, where $E_{\mathrm{V}}$ is the top of the valence band. Its dispersion follows closely the dispersion of the conduction band (see Fig. 4a). One can conclude that oxygen on a nitrogen site is a shallow donor in GaN SWNT like in the bulk GaN. With respect to the conduction band edge, the calculated ionization energy for $\mathrm{O}_{\mathrm{N}}$ is about $0.45 \mathrm{eV}$. This value is deeper than that of the wurtzite bulk GaN by about $0.32 \mathrm{eV}$ [14]. This makes it a suitable $n$-type dopant of GaN SWNT in high temperature uses.

\subsection{Zinc substitutional in GaN SWNT}

Zinc is the most investigated potential $p$-type dopant for bulk GaN. Zn impurity effectively compensates GaN resulting in high resistivity material [15-17]. Several groups [18-20] have published the most complete optical studies of the properties of Zn-doped GaN. All samples exhibited the commonly observed 2.8 $\mathrm{eV}$ emission. Both groups concluded that this peak corresponds to $\mathrm{Zn}_{\mathrm{Ga}}$ single acceptor center. Pankove and Berkeyheiser [21, 22] observed that Zn, present in sufficient concentrations, favors the substitutional replacements of $\mathrm{N}$ vacancies. Starting with these observations, we considered the zinc at both gallium and nitrogen sites. The substitutional replacement of Ga atom was achieved following the same procedure as discussed for oxygen resulting in two kinds of configurations: (i) Zn on an outer shell Ga site (configuration Zn-out); (ii) Zn on an inner shell Ga site (configuration Zn-in). After relaxation, it was found that the configuration $\mathrm{Zn}$-out is slightly $0.170 \mathrm{eV}$ lower in energy than the configuration Zn-in. The relaxation is tiny for this defect and the zinc atom moves inward a bit from the lattice site (see Fig. 3b). For the ground state configuration Zn-out, the Zn-N bond length is $1.904 \AA$ which is considerably shorter than the Ga-N bond length in the bulk. Mulliken charge analysis shows that the zinc is strongly positively ionized. $\mathrm{Zn}_{\mathrm{Ga}}$ is an acceptor as well in GaN SWNT as in bulk GaN. The defect 
level of the unpaired electron in our calculations is at about $E_{\mathrm{V}}+0.04 \mathrm{eV}$ (see Fig. 4b). This state is valence band derived. The acceptor level is at about 0.18 $\mathrm{eV}$ above the valence band edge, i.e. at $E_{\mathrm{V}}+0.18 \mathrm{eV}$. This indicates that $\mathrm{Zn}_{\mathrm{Ga}}$ is a shallow acceptor.

The zinc at the nitrogen site has been also studied. Zn on an innermost shell $\mathrm{N}$ site (configuration Zn-in) was found to be the ground state lying $0.257 \mathrm{eV}$ lower in energy than the configuration Zn-out. It is important to note that the relaxation for this defect is quite large. In the ground state, zinc moves deeply inward from the lattice site. This effect is more pronounced than for $\mathrm{Zn}_{\mathrm{Ga}}$. The $\mathrm{Zn}-\mathrm{Ga}$ bond length is about $2.470 \AA$ which is considerably larger than the Ga-N bond length in the bulk. Mulliken charge analysis shows that the zinc is slightly positively ionized. $\mathrm{Zn}_{\mathrm{N}}$ is an acceptor as well in GaN SWNT as in bulk GaN. In bulk GaN, a substitutional $\mathrm{Zn}$ on an $\mathrm{N}$ site might be capable of binding up to three electrons forming deep acceptor-like centers [23]. In GaN SWNT, our calculations show clearly that $\mathrm{Zn}_{\mathrm{N}}$ introduces a set of acceptor states ranging from 0.24 to 2.29 $\mathrm{eV}$ above the valence band edge (see Fig. 4c). The defect level around $E_{\mathrm{V}}+0.24$ $\mathrm{eV}$ falls deeply into the band gap. The deep acceptor level is at about 1.27 above the valence band edge, i.e. at $E_{\mathrm{V}}+1.27 \mathrm{eV}$. This indicates that $\mathrm{Zn}_{\mathrm{N}}$ can act as a compensating center.

While $\mathrm{Zn}_{\mathrm{Ga}}$ and $\mathrm{Zn}_{\mathrm{N}}$ are both acceptors in GaN SWNT, it is judicious to know which the preferred configuration is. To do this, we calculated the formation energy $\left(E_{\text {form }}\right)$ of these defects. This parameter is the energy needed to create a defect. It is important to assess the likelihood of their incorporation during growth and processing. DFT calculations are known to underestimate the semiconductor band gaps. Here the calculated gap is found to be $2.35 \mathrm{eV}$ and the error does not influence the formation energies of neutral defects, but may strongly affect those of the charged defects. We note straightaway that we are not interested in the precise values of the absolute formation energies but we rather want to compare the stabilities of structures. The formation energy of a structure with impurity in a neutral state is given as follows:

$$
E_{\text {form }}=E_{\text {total }}-n_{\mathrm{Ga}} \mu_{\mathrm{Ga}}-n_{\mathrm{N}} \mu_{\mathrm{N}}-\mu_{\mathrm{I}} \text {, }
$$

where $E_{\text {total }}$ is the total energy of a structure with impurity, $n_{\mathrm{Ga}}$ and $n_{\mathrm{N}}$ are the numbers of $\mathrm{Ga}$ and $\mathrm{N}, \mu_{\mathrm{Ga}}, \mu_{\mathrm{N}}$ and $\mu_{\mathrm{I}}$ are the chemical potentials of $\mathrm{Ga}$, $\mathrm{N}$, and the impurity ( $\mathrm{O}$ or $\mathrm{Zn})$ respectively. In compound semiconductors the chemical potential of the host constituents depends on the stoichiometry during growth and we consider the two limiting cases of Ga-rich and N- rich conditions. In GaN SWNT, $\mu_{\mathrm{Ga}}$ and $\mu_{\mathrm{N}}$ are dependent quantities through the relationship $\mu_{\mathrm{GaN}(\mathrm{SWNT})}=\mu_{\mathrm{Ga}}+\mu_{\mathrm{N}}$. Under Ga-rich conditions, $\mu_{\mathrm{Ga}}$ can be obtained from total energy calculations as $\mu_{\mathrm{Ga}}$ (bulk) and $\mu_{\mathrm{N}}$ is set equal to $\mu_{\mathrm{GaN}(\mathrm{SWNT})}-\mu_{\mathrm{Ga}}$. Under N-rich conditions, $\mu_{\mathrm{N}}$ is taken as the half of the calculated total energy of $\mathrm{N}_{2}$ molecule and $\mu_{\mathrm{Ga}}$ is set equal to $\mu_{\mathrm{GaN}(\mathrm{SWNT})}-\mu_{\mathrm{N}}$. We first calculated the energy difference of the formation energies of $\mathrm{Zn}_{\mathrm{Ga}}$ between the limiting cases of 
Ga-rich and N-rich conditions

$$
\Delta E\left(\mathrm{Zn}_{\mathrm{Ga}}\right)=E_{\text {form }}^{\mathrm{Ga}-\text { rich }}\left(\mathrm{Zn}_{\mathrm{Ga}}\right)-E_{\text {form }}^{\mathrm{N}-\text { rich }}\left(\mathrm{Zn}_{\mathrm{Ga}}\right)=-\Delta H_{\mathrm{f}} .
$$

Like in bulk GaN polytypes, the heat of formation of GaN SWNT is negative; therefore, $\Delta E\left(\mathrm{Zn}_{\mathrm{Ga}}\right)$ is positive. This means that the formation of $\mathrm{Zn}_{\mathrm{Ga}}$ is favorable under the N-rich conditions. The difference between the formation energies of $\mathrm{Zn}_{\mathrm{N}}$ between the extreme Ga-rich and N-rich limits has been calculated as follows:

$$
\Delta E\left(\mathrm{Zn}_{\mathrm{N}}\right)=E_{\text {form }}^{\mathrm{Ga}-\text { rich }}\left(\mathrm{Zn}_{\mathrm{N}}\right)-E_{\text {form }}^{\mathrm{N}-\text { rich }}\left(\mathrm{Zn}_{\mathrm{N}}\right)=\Delta H_{\mathrm{f}} .
$$

Since the heat of formation of GaN SWNT is negative, the formation of $Z_{N}$ will be favorable under the Ga-rich condition like in wurtzite bulk GaN. We also calculated the difference between the formation energies of $\mathrm{Zn}_{\mathrm{Ga}}$ and $\mathrm{Zn}_{\mathrm{N}}$ :

$$
\Delta E(\mathrm{Zn}) \Delta E_{\text {total }}(\mathrm{Zn})+\mu_{\mathrm{Ga}}-\mu_{\mathrm{N}} .
$$

$\Delta E(\mathrm{Zn})$ is the total energy difference of $\mathrm{Zn}_{\mathrm{Ga}}$ and $\mathrm{Zn}_{\mathrm{N}}$ in GaN SWNT. In Garich conditions, Eq. (5) yields about $0.14 \mathrm{eV}$ while in extreme N-rich condition it is about $-0.58 \mathrm{eV}$. This means that $\mathrm{ZnGa}$ is more favorable in N-rich and $\mathrm{ZnN}$ is preferentially formed near the Ga-rich region. In the stoichiometry $\left(\mu_{\mathrm{Ga}}=\mu_{\mathrm{Ga} \text { (bulk) }}-\Delta H_{\mathrm{f}} / 2\right)$, Eq. (5) yields about $-0.36 \mathrm{eV}$. Close to this limit, $\mathrm{Zn}_{\mathrm{Ga}}$ preferentially forms. Finally, we calculated the ionization energy for $\mathrm{Z} \mathrm{n}_{\mathrm{Ga}}$. With respect to the valence band edge our result is about $0.2 \mathrm{eV}$. In the bulk $\mathrm{GaN}$, the experimental ionization energy associated with $\mathrm{Zn}$ is estimated to be $0.34 \mathrm{eV}[18,24]$. This value is relatively larger; therefore, $\mathrm{Zn}$ doping seems to be inefficient. The main reason for this is presumably because the large acceptor ionization energy leads to hole concentrations about two orders of magnitude smaller than the dopant concentration at room temperature. In GaN SWNT, the calculated ionization energy for $\mathrm{Zn}_{\mathrm{Ga}}$ is about $200 \mathrm{meV}$. This value is relatively smaller than its counterpart in the wurtzite bulk structure. This suggests that $\mathrm{Zn}_{\mathrm{Ga}}$ can be employed in the $p$-doping of GaN nanotubes. However, the performance of an acceptor cannot be judged only on the depth of its ionization energy but also on its solubility and stability against compensation by other configurations. Close to the stoichiometry, both $\mathrm{Zn}_{\mathrm{Ga}}$ and $\mathrm{Zn}_{\mathrm{N}}$ form, although $\mathrm{Zn}_{\mathrm{Ga}}$ is in greater extent, thus suggesting a shallow-deep transition as the source of the drop in hole concentration achievable with $\mathrm{Zn}$. In the extreme $\mathrm{N}$-rich condition, $\mathrm{Zn}_{\mathrm{Ga}}$ will be the most favorable configuration. Therefore, if we consider that the $\mathrm{Zn}$ does not suffer from incorporation on sites other than the substitutional ones, Zn may be an efficient $p$-type dopant of GaN SWNT.

\section{Summary}

In summary, we have studied the structure and electronic properties of substitutional zinc and oxygen impurities in a model of single wall GaN nanotubes by means of ab initio supercell calculations. We found that oxygen forms a shallow donor in GaN SWNT like in bulk GaN polytypes. The deep ionization energy 
associated with $\mathrm{O}_{\mathrm{N}}$ makes it a suitable $n$-type dopant in high temperature applications. Zinc is a relatively shallow acceptor at gallium site while at nitrogen site it is a charge carrier trap. The site preference strongly depends on the stoichiometry of GaN SWNT. The calculated ionization energy for $\mathrm{Zn}_{\mathrm{Ga}}$ is relatively smaller, thus it would be an efficient $p$-dopant in GaN nanotubes unlike in bulk GaN polytypes. If the GaN SWNT is stabilized, the control of the concentration and the site selection of zinc would have important consequences in technological applications.

\section{References}

[1] S. Nakamura, T. Mukai, M. Senoh, Appl. Phys. Lett. 64, 1687 (1994).

[2] J. Goldberger, R. He, Y. Zhang, S. Lee, H. Yan, H.-J. Choi, P. Yang, Nature (London) 422, 599 (2003)

[3] S.M. Lee, Y.H. Lee, Y.G. Hwang, J. Elsner, D. Porezag, T. Frauenheim, Phys. Rev. B 60, 7788 (1999).

[4] S. Iijima, Nature (London) 354, 56 (1991).

[5] A. Gali, Phys. Rev. B 73, 245415 (2006).

[6] J.M. Soler, J.D. Gale, A. García, J. Junquera, P. Ordejón, D. Sanchéz-Portal, J. Phys. Condens. Matter 14, 2745 (2002).

[7] J.P. Perdew, K. Burke, Mathias Ernzerhof, Phys. Rev. Lett. 77, 3865 (1996).

[8] N. Troullier J.L. Martins, Phys. Rev. B 43, 1993 (1991).

[9] Q. Wang, Q. Sun, P. Jena, Y. Kawazoe, Phys. Rev. B 73, 205320 (2006).

[10] H.J. Monkhorst J.K. Pack, Phys. Rev. B 13, 5188 (1976).

[11] M. Zhang, Z.-M. Su, L.-K. Yan, Y.-Q. Qiu, G.-H. Chen, R.-S. Wang, Chem. Phys. Lett. 408, 145 (2005).

[12] CRC Handbook of Chemistry and Physics, 67 ed., Ed. by R.C. West, CRC, Boca Raton FL 1986.

[13] J. Neugebauer, C.G. Van de Walle, Festkörperprobleme/Adv. Solid State Phys. 35, 25 (1996).

[14] C. Liu, J. Kang, Optical Materials 23, 169 (1903).

[15] H.P. Maruska, J.J. Tietjen, Appl. Phys. Lett. 15, 327 (1969)).

[16] E. Ejder, P.-O. Fagerstrm, Phys. Chem. Solids 36, 289 (1975).

[17] R.K. Crouch. W.J. Debnam, A.L. Fripp, J. Mater. Sci. 13, 2358 (1978).

[18] B. Monemar, O. Lagerstedt, H.P. Gislason, J. Appl. Phys. 51, 625 (1980).

[19] G. Jacob, M. Boulou, M. Furtado, J. Cryst. Growth 42, 136 (1977).

[20] M. Boulou, M. Furtado, G. Jacob, D. Bois, J. Lumin. 18/19, 767 (1979).

[21] J.I. Pankove, J.E. Berkeyheiser, E.A. Miller, J. Appl. Phys. 45, 1280 (1974).

[22] J.I. Pankove, J.E. Berkeyheiser, J. Appl. Phys. 45, 3892 (1974).

[23] I. Gorczyca, A. Svane, N.E. Christensen, Solid State Commun. 101, 747 (1997).

[24] S. Strite, H. Marko, J. Vac. Sci. Technol. B 10, 1237 (1992); S. Strite, in: Properties of Group III Nitrides, Ed. J.H. Edgar, INSPEC, Kansas City 1996, p. 272. 